\title{
CONCEPTUAL DESIGN OF SUPERCONDUCTING QUADRUPOLE ARRAYS FOR HEAVY-ION FUSION *
}

\author{
R. B. Meinke ${ }^{\#}$, Advanced Magnet Lab, Inc. Palm Bay, FL \\ A. Faltens, R. O. Bangerter, Lawrence Berkeley National Lab, Berkeley, CA
}

\begin{abstract}
Extensive studies have been performed to optimize the design of superconducting quadrupole arrays for beam transport in future heavy-ion fusion accelerators. In these arrays 20 or more quadrupole coils are densely packed with their axes aligned in parallel. Field strengths between 3 and 5 tesla at the inner coil diameters have been investigated. The aperture of the individual quadrupoles has been varied between 60 and $240 \mathrm{~mm}$. The coils have a typical length of $1 \mathrm{~m}$, and special effort has been made to minimize the length of the interconnect region between the array and adjacent accelerator components. The relative orientation of quadrupoles in the array is chosen to optimize field sharing between neighboring cells. Field uniformity in the boundary cells is maintained by placing concentric correction coils onto these cells. A conceptual cryostat design, which minimizes heat loads on the cryogenic system, has been analyzed. Since Inertial Fusion Energy has to compete with other long-term energy sources, cost and reliability of these magnets are major issues. The presented design uses a round NbTi minicable, which allows for a cost-effective, highly automated manufacturing of the required superconducting coils.
\end{abstract}

\section{INTRODUCTION}

Conventional accelerator technology meets the requirements of drivers for inertial fusion energy production. Existing accelerators operate reliably 24 hours/day with lifetimes of more than 40 years in densely populated areas and yield high pulse repetition rates with high efficiency. Beams in accelerators are transported in high vacuum, using magnetic dipoles for beam steering and quadrupoles for focusing. For fusion, the beam current for a given aperture and quadrupole strength is limited more by space charge effects than by emittance. The necessary beam currents for fusion drivers can only be achieved by using 20 or more beams in parallel [1]. Beam transport in such an accelerator with many parallel beams requires arrays of quadrupoles, which furnish the necessary number of parallel focusing channels.

\footnotetext{
* This work was supported in part by U.S. Department of Energy under contract No. DE-AC03-76SF00098.

"Email: rmeinke@magnetlab.com
}

The work presented in this paper addresses these issues for the superconducting quadrupole arrays. An array has been designed that meets the technical specifications of a fusion driver. The individual quadrupole cell is valueengineered by considering all costs from materials to manufacturing technology. The developed design is scalable over an inner coil diameter (ID) from 60 to $240 \mathrm{~mm}$ and in field strength at the coil ID from 3 to 5 tesla. A cost model developed for this design can be used in a global accelerator optimization to find the most costeffective parameters of the quadrupoles.

Using the value-engineered quadrupole, an array has been designed that optimizes flux sharing between the individual cells. Required field quality in the boundary cells of the array is achieved with the help of small correction coils that are directly wound onto these outer cells. A conceptual design of the cryostat has been produced, which guarantees good relative alignment of the quadrupoles and has low heat loads at $4.2 \mathrm{~K}$.

\section{INDIVIDUAL QUADRUPOLE CELL}

\subsection{Superconductor}

The coils are wound from a NbTi round mini-cable with 6 around 1 strands. A round cable is less expensive than a Rutherford type cable, shows no significant degradation in critical current density due to the cabling process, and is easier to handle in coil winding since it can be bent easily in any direction. The parameters related to the cable are listed in Table 1.

Table 1: Cable Parameters

\begin{tabular}{|l|c|c|}
\hline \multicolumn{1}{|c|}{ Item } & Value & Unit \\
\hline Number of Strands & 7 & \\
\hline Strand Diameter & 0.65 & $\mathrm{~mm}$ \\
\hline Cable Diameter & $\sim 2$ & $\mathrm{~mm}$ \\
\hline Cu/SC ratio & $2.5 / 1$ & \\
\hline $\begin{array}{l}\text { Critical Current @ } \\
\text { 4.2 K, 5 tesla }\end{array}$ & 3,100 & $\mathrm{~A} / \mathrm{mm}^{2}$ \\
\hline Insulation & Kapton wrap & \\
\hline
\end{tabular}

In the accelerator the quadrupole arrays will not be adjacent to each other, and the magnet current has to be routed through an external transfer line to the next magnet. It is therefore advantageous to operate the magnets at 
a relatively low current. The round mini-cable has the additional advantage that the splices between the individual coils of the array are easier to make and require less space than would be needed for a Rutherford type cable. This is of particular importance for the quad arrays since only about $250 \mathrm{~mm}$ are available at both ends of the magnet for the connection to adjacent accelerator components.

The operational parameters of the magnet are summarized in Table 2.

Table 2: Operational Parameters

\begin{tabular}{|l|c|c|}
\hline \multicolumn{1}{|c|}{ Item } & Value & Unit \\
\hline Nominal Current & 2,366 & $\mathrm{~A}$ \\
\hline Safety Margin & 20 & $\%$ \\
\hline Operational Temp. & 4.35 & $\mathrm{~K}$ \\
\hline Critical Temp. & 4.78 & $\mathrm{~K}$ \\
\hline Current Density in $\mathrm{Cu}$ & 1,200 & $\mathrm{~A} / \mathrm{mm}^{2}$ \\
\hline
\end{tabular}

With a strand diameter of $0.65 \mathrm{~mm}$, the cable diameter is about $2 \mathrm{~mm}$. It is assumed that the critical current of the conductor is $3,100 \mathrm{~A}$ at $4.2 \mathrm{~K}$ and 5 tesla, which is the best conductor currently available. The nominal current is 2,366 A.

\subsection{Coil Design}

A cross section that is typical for all considered quadrupoles is shown in Figure 1. All coils consist of 5 to 9 layers of conductor, depending on their diameter and field strength.

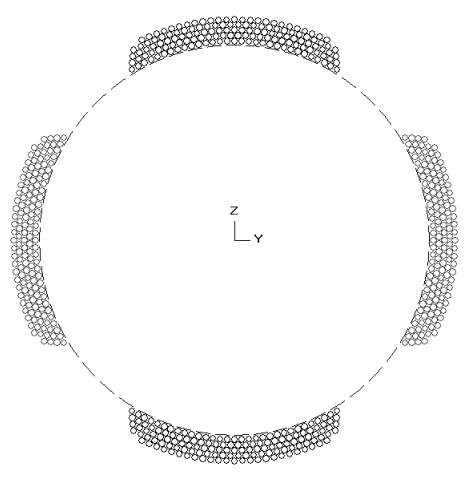

Figure 1: Coil cross section of 4-tesla, 120-mm diameter quadrupole.

The coil is wound onto a fiber-reinforced composite structure (see Figure 2), which rests on a stainless steel (SS) support tube. The coil is totally embedded into a fiber-reinforced composite and then surrounded by an aluminum (Al) shrink cylinder. This structure has sufficient stability to counteract the Lorentz forces during coil excitation in radial and axial direction of the coil.

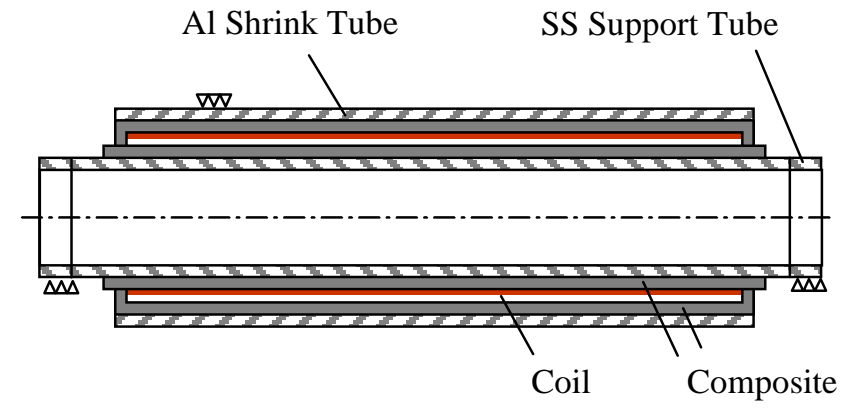

Figure 2: Layout of individual quadrupole coil

The ends of the SS tube are machined to be concentric to each other, and the outer surface of the Al cylinder is machined to be concentric with these ends. These machined surfaces guarantee a precise alignment of the coils in the array.

The coils are wound with an automatic winding machine, which allows placing the round mini-cable into machined pockets in the composite structure. Adhesive for conductor bonding inside of the coil is automatically added during the winding process. The computer-controlled winding process does not only guarantee precise conductor placement, but also yields a very inexpensive manufacturing process of coils with excellent performance [2].

\section{ARRAY LAYOUT}

The individual coils are assembled to form an array of quadrupoles. A section of such an array with the individual cell orientation is shown in Figure 3.

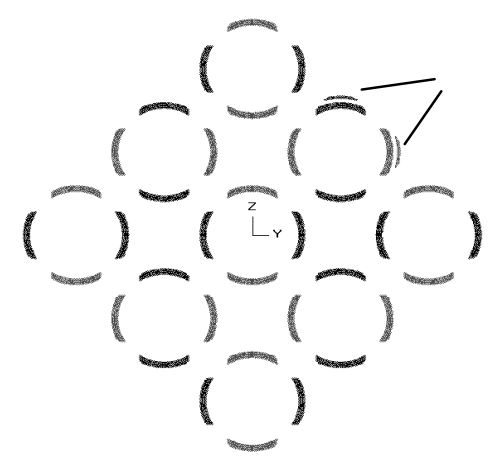

Figure 3: Arrangement of cells in the array. One boundary cell equipped with correction coils.

\subsection{Flux Sharing}

Since the coils are in close proximity to each other flux penetrates into neighboring cells. The relative orientation, indicated in Figure 3, enhances the flux in neighboring cells, and about $30 \%$ of the fields in any cell are due to flux from its neighbors. The cells in this arrangement 
yield both, horizontal and vertical focusing, which can be accommodated in the accelerator lattice. Because the channels are dependent on each other, a completely separate lower-field iron core array consisting of correction coils is required for independent beam steering.

\subsection{Edge Termination}

Cells at the array boundaries have no neighbors on one side and a strong dipole field component results. This could be corrected with dummy cells at the edge of the array. A more cost-effective edge termination scheme is indicated for one of the boundary cells in Figure 3. A small correction coil wound with the same conductor and in series with the main coil totally corrects the field in this cell.

A superconducting shield, which surrounds the whole array at a certain distance, is under consideration. Persistent currents induced into this passive shield would automatically arrange themselves in such a way that the flux at the outside of the shield is zero. This shield would eliminate any magnetic effect on neighboring accelerator components.

\section{CRYOSTAT}

\subsection{Cell Support}

The individual quadrupole coils are held in place by large aluminum carrier plates. As indicated in Figure 4 the individual coils fit into precisely machined holes in the carrier plates. The $10-\mathrm{mm}$ thick plates are spaced with gaps of $75 \mathrm{~mm}$ width along the axes of the coils. The plates hold and align the individual coils, and strengthen the $\mathrm{Al}$ shrink cylinders. SS alignment keys position the carrier plates relative to each other.

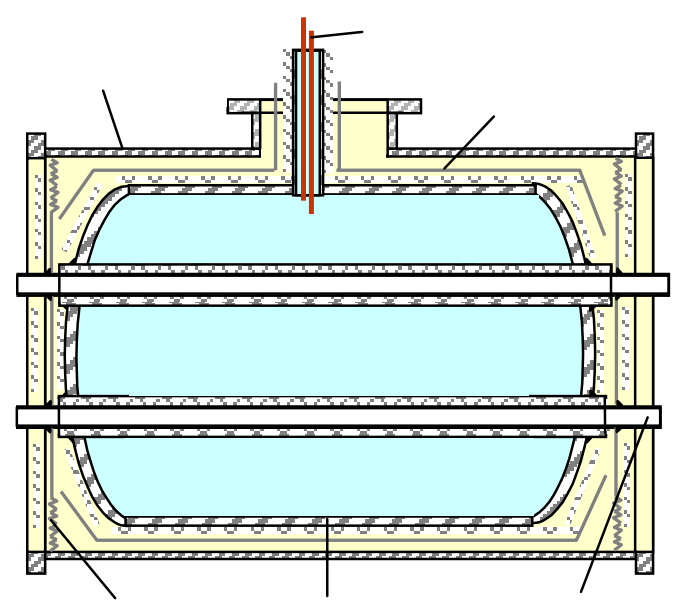

Figure 4: Schematic layout of cryostat. For reasons of clarity, only two beam tubes are shown.
$\mathrm{Al}$ is chosen as a material for the shrink cylinders and the carrier plates because of its thermal contraction, which is larger than SS. During cool down of the coils the Al shrinks more than the SS support tubes and puts the superconducting coils under compression.

An SS cylinder around the carrier plates forms the helium containment vessel. This cylinder is closed at both ends with SS end plates, which are welded helium-tight to the SS support tubes. Due to the strengthening by the SS tubes, thin straight end plates are sufficient for this pressure vessel, which has to take the overpressure with sufficient safety margin from gaseous helium during a quench. The straight plates help to reduce the total length of the array.

A radiation shield and blankets of superinsulation surround the helium containment vessel. Struts are used as a support inside of the outer vacuum vessel, which is evacuated to achieve thermal insulation.

\subsection{Beam Tube}

Beam tubes are inserted into the SS coil support tubes to maintain low residual gas pressure for beam transport. The beam tubes have to be operated at $77 \mathrm{~K}$, the temperature of the thermal shield, to reduce their heat load into the superconducting coil to an acceptable level. With a beam tube polished on the outside and the SS support tube on the inside no superinsulation is required, and the heat load due to thermal radiation from up to 50 beam tubes is less than 1 watt.

\section{SUMMARY}

Superconducting quadrupole arrays for a future heavy ion fusion accelerator have been designed. The scalable coil design allows variations of aperture from 60 to $240 \mathrm{~mm}$ and fields at the coil ID from 3 to 5 tesla. The novel coil design significantly reduces cost in comparison to conventional collared coils made from Rutherford type cable. A cost model has been developed that allows one to identify the most cost effective parameters for the quadrupole array. A preliminary design of the array cryostat has been developed that minimizes space requirements along the array axis and has heat loads comparable to existing highenergy physics accelerators.

\section{REFERENCES}

[1] Proceedings of 12th International Symposium on Heavy Ion Inertial Fusion, Heidelberg, Germany, September 1997, Nuclear Instruments \& Methods in Physics Research (Section A) - VoL. 415 (1998) Nos. 1,2, pp. 1-508.

[2] R.B. Meinke et al., "Sextupole Correction Magnets for the Large Hadron Collider", submitted to this conference. 